\title{
Unique Mechanisms for the Availability of Declarative Memory Elements and the Strengthening of Cognitive Operations
}

\author{
Dale A. Hirsch', Christopher A. Was', and Erin N. Graham² \\ ' Naval Service Training Command, Great Lakes, IL \\ 2 Department of Psychological Sciences, Kent State University
}

ABSTRACT

The purpose of this study was to investigate the role of memory for prior cognitive operations and availability of declarative memory elements in long-term semantic priming. The impetus for this investigation was the role of working memory (WM) in complex cognitive processing. Empirical estimates of WM are too limited to explain complex cognitive processes. Therefore, contemporary models of WM propose access to long-term memory (LTM) to expand these limits. The priming literature provides one theoretical mechanism for access to LTM: long-term semantic priming. However, explanations for long-term semantic priming include both increased availability of LTM elements and the facilitation of prior cognitive operations. Our goal was to examine if the facilitation of prior cognitive operations is dependent on the availability of previously encountered LTM elements. A task used in previous research proposed to capture the facilitation of cognitive operations coupled with a directed forgetting manipulation was used to examine this relationship. Three experiments were conducted to that end. All experiments resulted in facilitation of the procedure of categorization. Experiments 1 and 2 additionally found relatively poor recognition for items that participants were told to forget despite the fact that categorization was facilitated for related items. Experiment 3 resulted in similarly poor recognition for category names that participants were told to forget. Taken together, the experiments in this investigation demonstrate a clear separation between the cognitive operations and declarative elements of the categorization task. Namely, the continued availability of declarative elements is not necessary for the subsequent facilitation of

categorization operations.

\author{
KEYWORDS \\ long-term semantic priming \\ working memory \\ available long-term memory \\ priming \\ directed forgetting
}

\section{INTRODUCTION}

Working memory (WM), as described by many models, is a limited cognitive workspace. To accommodate for the empirically derived limits of WM-that are far too limited to describe complex cognitive processing several models of WM describe the contents of this cognitive workspace in terms of long-term memory (LTM) elements that are available for processing due to an increased level of activation (e.g., Anderson, 1993; Cowan, 1995, 1999; Just \& Carpenter, 1992; Oberauer, 2002). Activated LTM elements include those activated to a degree that allows for attentional focus and processing, but also includes elements with less activation and outside of attentional focus, yet in a state of relative readiness for processing. The WM models of Cowan $(1995,1999)$ and Oberauer $(2002,2009)$ make an explicit distinction between attention-based WM and activated LTM components. Cowan's embedded processes model distinguishes between those memory elements in the focus of attention, memory elements that were activated and available for processing although they were not in focus of attention, and all other memory elements that are relatively inactive and thus not available. Oberauer has also proposed and presented empirical evidence for the concentric model that includes differing levels of LTM memory element activation.

To examine whether processing of information in the focus of attention would lead to increased availability of related but unattended to information, Woltz and Was (2006) developed the availability of long-term

Corresponding author: Christopher A. Was, Department of Psychological Sciences, Kent State University, 144 Kent Hall, Kent, OH 44303.

E-mail: cwas@kent.edu 
memory (ALTM) task. The impetus for the series of experiments by Woltz and Was $(2006,2007)$ was the assumption that attention-driven processing in WM leads to the activation of declarative LTM elements related to those that were previously processed. This is in concordance with the WM model proposed by Oberauer (2009) and Cowan's (1999) embedded processes model. Although their goal was to determine if simple processing in WM would lead to the activation of related, but not processed declarative memory elements, their work eventually led to examination of the memory for prior cognitive operations

The original experimental task (Woltz \& Was, 2006; Experiment 1) included four distinct components. The first was a memory load consisting of exemplars from two distinct categories which participants were to maintain in WM (e.g., "oak," "table," "elm," "chair”). The second component was an instruction to focus on some of the specific items presented (e.g., "Remember the trees"). Next, the participants were asked to recall the to-be-remembered items (e.g., "What were the words you were to remember?"). Finally, participants were asked to make a series of category comparison judgments. Each comparison trial presented two words at a time and required the participants to determine if the two words belonged to the same semantic category. Half of the comparisons were negative matches whereas the other half were positive matches. The words used in the comparisons came from the remembered category (e.g., "pine"-"fir"), the ignored category (e.g., "sofa"-“ottoman"), or from a neutral category (e.g., "terrier"- “spaniel”). Remembered and ignored exemplars in the comparison task belonged to the memory load categories (i.e., "trees" and "furniture" in this example) but were not the exemplars from the memory load. Results of each of the experiments indicated that participants were faster and more accurate at determining if two exemplars were from the same category when the category comparison stimuli were from either category (remember and ignor) presented in the memory load compared to a neutral (not previously presented) category. Notably, participants were also significantly faster and more accurate in their responses to the remember than the ignore category comparison. The results of these experiments beg the question of the underlying mechanism responsible for the response facilitation demonstrated in the ALTM task. One possible explanation is that the processing during the memory load and category-focused parts of the task increased the availability of the declarative element(s) and word associations. Alternatively, it may be that the cognitive processing required in the ALTM task strengthens the cognitive operations relevant to the task (e.g., identification of category membership). Therefore, it is reasonable to suspect that the results of Experiment 1 from Woltz and Was (2006) could be the result of increased availability of declarative memory elements, the strengthening of prior cognitive operations, or some degree of both.

Insight regarding the mechanisms responsible for the facilitation effects demonstrated by Woltz and Was $(2006,2007)$ is found in the semantic priming literature. Semantic priming is defined as the facilitation of response speed and/or accuracy to a target item that occurs as a result of the presentation of a semantically related image or word (the prime) compared to the response to an unrelated image or word (McNamara, 2005). For example, in an experimental task the presentation of the word "fork" facilitates better access to or decisions regarding "spoon" compared to "doctor." Several models of semantic priming have been proposed to account for semantic priming, such as spreading activation models (Collins \& Loftus, 1975; Quillian, 1967) and compound-cue models (Dosher \& Rosedale, 1989; Ratcliff \& McKoon, 1988), each of which describes the facilitation effects of semantic priming as being due to the increased availability of declarative memory elements. Regardless of the explanation of semantic priming, early models of semantic priming held the view that the effects were short-lived, typically lasting a few seconds or across one intervening experimental trial. These models have been updated and replaced as a substantial corpus of literature compiled over more than thirty years has established a clear distinction between short-term and long-term semantic priming (facilitation effects over longer time spans). Originally, it was theorized that a single mechanism was responsible for both short and long-term priming. Later models refuted this explanation and proposed that different mechanisms are necessary to account for different durations of priming effects. Two alternative explanations of long-term semantic priming effects have been proposed in the extant literature. The first explanation is that longterm semantic priming effects rely upon the incremental strengthening of abstract semantic memory representations. The second explanation is that long-term priming is the result of memory for prior cognitive operations.

Becker et al. (1997) and Joordens and Becker (1997) first demonstrated long-term semantic priming effects lasting several seconds and with several intervening trials. Importantly, their evidence demonstrated priming effects at longer lags than could be explained by then current theoretical models of short-term priming such as spreading activation (McNamara, 1992), compound cues (Dosher \& Rosedale, 1989; Ratcliff $\&$ McKoon, 1988), and distributed networks (Masson, 1995). They suggested that greater semantic processing demands in their priming tasks accounted for the novel empirical evidence and proposed a distributed network model that incorporated persistent rather than temporary network changes from prime processing.

The initial network model proposed by Becker et al. (1997) contained a single mechanism to explain priming effects at short and long lags. In contrast, follow-up work by Joordens and Becker (1997) suggested that different model components were necessary to account for short- and long-term priming effects. This proposed distinction relates to a subsequent discussion by McNamara (2005), who questioned whether extant long-term priming evidence represented memory processes that differed from those underlying temporary semantic priming effects found in lexical decision and naming experiments.

In their series of studies, Woltz and Was $(2006,2007)$ conducted two experiments most relevant to explanations of long-term priming and the current investigation. Experiment 2 in Woltz and Was (2007) explored the mechanism by which the target category comparisons were facilitated to examine whether increased availability of declarative memory elements or the strengthening of prior cognitive operations was responsible for their previous findings. To investigate these ideas, Woltz and Was (2007) used the same experimental procedure as Woltz and Was (2006), but introduced a new stimulus type to the task: category 
features. A feature is a component or attribute of a broader semantic category. For example, the features "wing", "feather", and "beak" belong to the semantic category "bird". Experiment 2 from Woltz and Was (2007) altered their original 2006 paradigm by ensuring that the memory load contained either all exemplars or all features from two semantic categories. For example, in one trial, the participant may have seen a memory load consisting of features from the "bird" and "sports" categories (e.g., "wing," "racket," "feather," and "ball"). Similarly, the comparison phase contained either all exemplars (e.g., "robin"-"cardinal") or all features (e.g., "talon"-"beak"). This created a $2 \times 2$ (memory load type [exemplars or features] $\times$ comparison type [exemplars or features]) design. The results showed the facilitation effect for congruent trials (i.e., trials having exemplars or features in both the memory load and comparison phases), but no facilitation in trials that crossed stimulus type (i.e., exemplars and features) between the memory load and comparison phases. That is, when the memory load contained exemplars and subsequent comparisons were between new exemplars belonging to the memory load categories, the results replicated their previous findings.

The same pattern was replicated when a memory load of features was followed by a comparison phase using features. Importantly, facilitation was not present in trials that did not match stimulus types across trial phases. When exemplars were used in the memory load, category comparisons between features did not show facilitation. The same was true of exemplar comparisons following a memory load of features. Together, these results suggest that the processing involved in the ALTM task strengthens the cognitive operation of category identification differently for exemplars and features, and hence, they rely on different operations. Because the exemplar memory load facilitated exemplar comparisons but failed to facilitate feature comparisons from within the same category, it suggests that the cognitive operation of identifying exemplars of a category is distinct from that for identifying features of a category.

Others have reported similar results. Hughes and Whittlesea (2003) reported the results of a several experiments that suggested operation specificity in semantic and conceptual priming. They demonstrated that semantically mediated priming effects in specific target operations (e.g., naming, lexical decision, exemplar categorization, feature categorization) occurred only when the same operation from the target event was required in the priming event. Their findings were generally consistent with those of Woltz and Was (2007, Experiment 2).

Moreover, these results are inconsistent with an explanation based on the increased availability of declarative elements. If the mechanisms responsible for the increased availability of declarative elements were the same as those for categorization, one would expect facilitation for both related exemplars and related features due to the high semantic associations among these items. Instead, the lack of facilitation across related exemplars and features seems to indicate operational specificity between the congruent and noncongruent tasks.

The second experiment related to the current study is Experiment 2 in Woltz and Was (2006), which was designed to address the possible influence of explicit category labels on availability of relevant declarative memory elements. The procedure of Experiment 2 followed that of the basic ALTM paradigm (Woltz \& Was, 2006, Experiment 1, described above) with the exception that, in half of the trials, the focus instruction labeled the remember category (e.g., "remember the trees") and the other half of the trials labeled the ignore category (e.g." "ignore the furniture"). Only one of the categories from the memory load was explicitly labeled in each trial and the remember category was always recalled. The intention of the manipulation in Experiment 2 was to determine whether the findings of Experiment 1 (i.e., that the response facilitation of the categorization task was greater for the remember category as compared to the ignore category) were due to the processing of the category label itself or to the extra rehearsal that the to-be-remembered exemplars were likely to receive. The results of Experiment 2 showed that the largest response facilitation during the category comparison phase went to the category that was explicitly labeled. That is, during trials in which they were instructed to ignore a specific category, participants showed greater response facilitation for comparisons between exemplars of that ignored category. The same was true of remember category comparisons; for trials in which they were instructed to remember a specific category, participants showed greater response facilitation for comparisons between exemplars of that remembered category. Providing participants with the verbal label of the category had a larger effect upon the subsequent category comparison operation than did the extra rehearsal received by the remember category exemplars. This suggests that the operations involved in category comparison are not the same operations involved in maintaining the availability of the exemplars.

The results of Woltz and Was (2006, Experiment 2) provide evidence that the facilitation effects demonstrated in their ALTM task are, at least in part, due to the processing of the category labels. While intriguing, these results do not provide evidence that the facilitation of the category comparisons is independent of increased availability of all relevant declarative memory elements. Recall that on half of the experimental trials (Woltz \& Was, 2006, Experiment 2) participants were instructed to ignore a category during the memory load, yet participants were always asked to recall the exemplars they were to remember. Throughout the series of experiments, Woltz and Was $(2006,2007)$ never asked participants to recall the ignored exemplars. As such, there is no way to rule out the possible influence of increased availability of declarative memory elements on the facilitation of the category comparisons. It is possible that participants either actively maintained the ignored exemplars or that the exemplars retained some residual activation. If either is the case, then it is possible that the facilitation demonstrated in their work is at least partially dependent upon the availability of the relevant declarative memory elements and not due to the facilitation of the category comparison operation. The current study was designed to test the feasibility of this alternative explanation. Put differently, we hoped to further investigate whether the mechanism underlying the long-term priming effects in the exemplar comparison task is one of memory for prior cognitive operations or the availability of the category exemplars. To do so, we adapted the ATLM task used by Woltz and Was (2006, Experiment 2) and incorporated an item-method directed forgetting manipulation (Golding, 2005; Taylor et al., 2018).

In item-method directed forgetting experiments, participants are presented with items (typically words) one at a time and instructed 
to either remember or forget the items. In subsequent measures of memory for items (item recall or item recognition) participants perform less accurately on "forget" versus "remember" items (Taylor et al, 2018). The effects of item-method directed forgetting are assumed to occur at encoding (Taylor et al, 2018). If participants are instructed to remember an item, they are presumed to engage in rehearsal strategies to ensure later recall of the item. If they are instructed to forget an item, participants employ executive control to disengage attention from the item and thus prohibit rehearsal (Bastin et al, 2012; Hourihan \& Taylor, 2006; Taylor, 2005; Taylor \& Fawcett, 2011).

In the following three experiments, we added an item-method directed forgetting aspect to our design. In each experiment, participants were instructed to forget specific items and remember others. At the end of each experiment, participants were administered a recognition test of the exemplars (Experiments 1 and 2) or the categories (Experiment 3) that they encountered during the ALTM task. This allowed us to test our hypotheses regarding the mechanisms responsible for the long-term priming effects demonstrated in the ALTM task.

\section{EXPERIMENT 1}

\section{Participants}

To determine the number of participants, we conducted a power analysis using an effect size of $\eta^{2}=.17, f=.45$, an $\alpha$ of .05 , and power of .80. The effect size values were derived from Woltz and Was (2006). The effect size reflects the difference in facilitation effects between ignored category comparisons and neutral category comparisons in their Experiment 1 . We chose this effect size as our hypothesis is de- pendent on finding the same effect. The power analysis indicated that 32 participants would be sufficient. Thirty-six undergraduate students enrolled in an educational psychology course participated in the study for course credit. There were 6 male and 30 female participants. Their median age was 20 years old (range $=4$ years).

\section{Apparatus}

Participants completed the experimental task on IBM-compatible computers with SVGA monitors, standard keyboards, and circumaural sealed headphones. The experiment was programmed with E-Prime software (Schneider et al., 2002).

\section{Experimental Task}

Category stimuli for this experiment were adapted from earlier studies (Woltz \& Was, 2006, 2007). Though similar to the Woltz and Was (2006) experimental task, the details of the task components differed from the previous studies and included an item recognition task. Figure 1 presents an example of the order of the ALTM task components. All components of the ALTM task, with the exception of the memory load, were presented visually on the computer display. The four words in the memory load were presented aurally as they would later be presented visually in the recognition task. Cross-modality was applied as it was assumed this would eliminate facilitation of recognition from repeated perceptual processes and not contaminate the recognition task. Category stimuli were organized into 18 sets of three categories (i.e., remember, ignore, and neutral). These category triplets were organized to limit conceptual overlap among the three categories. For each participant, one category from each set was assigned to the remember category, one to the forget category, and one was assigned to
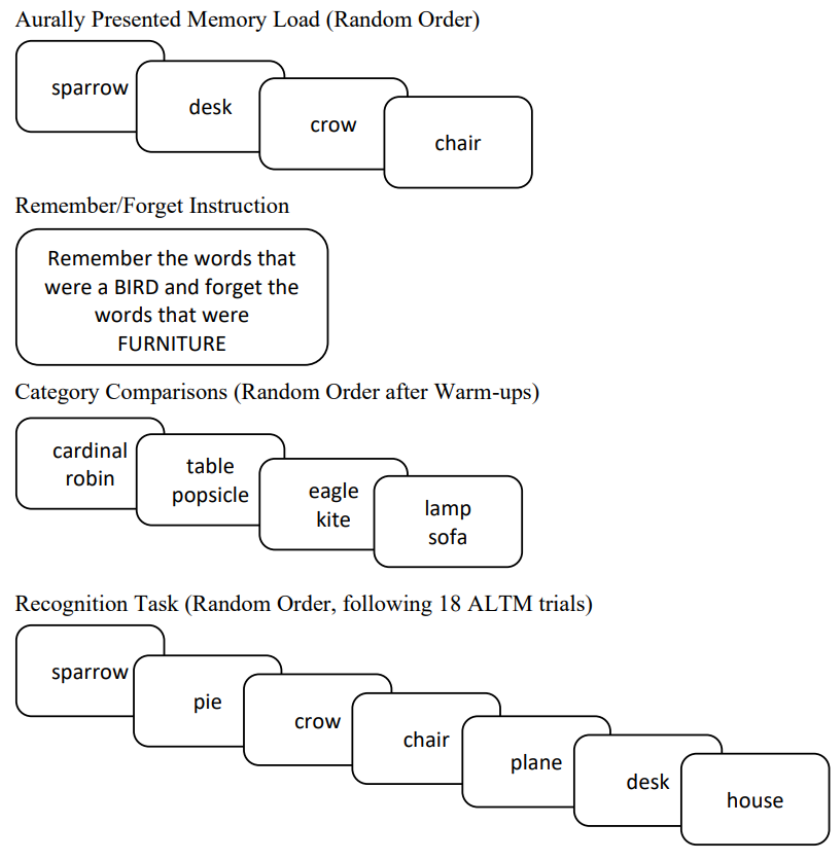

FIGURE 1.

- Example of the order of the ALTM task components. 
be a category not presented in the memory load (a neutral category). Six versions of the experiment were created to allow for complete counterbalancing of categories assigned to each of the facilitation conditions (i.e., remember, forget, and neutral).

\section{Availability of Long-Term Memory Task}

Participants completed 18 trials of the ALTM task portion of the experiment before completing the word recognition task. The components of the ALTM portion of the task (see Figure 1) are described first, then the recognition portion of the experiment. Each of the 18 ALTM trials used new stimuli to represent the remember, forget, and neutral stimuli described below.

\section{MEMORY LOAD}

The memory load contained four words, two each from two distinct categories. For example, one category might have been birds (e.g., "sparrow" and "robin") and the other two words furniture (e.g., "desk" and "chair"). Each memory load was preceded by the message "Get ready to MEMORIZE words" displayed on the monitor for $2 \mathrm{~s}$. A blank screen appeared for $1.5 \mathrm{~s}$, then a tone sounded for $1 \mathrm{~s}$ to focus the participants' attention, followed by an asterisk in the center of the screen for $500 \mathrm{~ms}$. The first word of the memory load was then presented. Each word was presented aurally over headphones. The words were presented one at time at a rate of $2 \mathrm{~s}$ per word. A $500 \mathrm{~ms}$ asterisk in the center of the screen preceded each word. The fourth and final word in the memory load was followed by a $3 \mathrm{~s}$ pause.

\section{REMEMBER/FORGET DIRECTION}

Following the $3 \mathrm{~s}$ pause after the presentation of the final memory load word, participants were instructed to both remember words from one category as well as forget the words from the other category. For example, the instruction might have read "Remember the words that were a BIRD. Forget the words that were FURNITURE." The instruction screen was self-paced and participants used the spacebar to proceed to the next screen. A $2 \mathrm{~s}$ pause followed the remember/forget instruction.

\section{CATEGORY COMPARISONS}

After the remember/forget instruction, participants completed 10 category comparisons. The category comparison phase began with a $3 \mathrm{~s}$ screen that read "Get ready to COMPARE words." Then, a $2 \mathrm{~s}$ blank screen allowed participants to prepare for the comparisons. Each category comparison frame began with a $2 \mathrm{~s}$ blank screen followed by a pair of asterisks appearing one above the other in the center of the screen for $500 \mathrm{~ms} .750 \mathrm{~ms}$ later the first category comparison appeared. Each category comparison presented two words at a time. The words were presented one above the other in the center of the screen where the asterisks had been. The participants were tasked with determining whether the two words belonged to the same semantic category. Participants were instructed to rest their index fingers on the " $D$ " and "L" keys. The "L" key was used to represent "like" comparisons and the
"D" key was used to represent "different" comparisons. The first four category comparisons were warm-ups and not used in data analysis. The final six comparisons were the actual trial comparisons. Of the six actual comparisons, three were like comparisons (i.e., the two words belonged to the same category) and three were different comparisons (i.e., the two words belonged to different categories). One of the like comparisons presented associates of the words belonging to the remember category from the memory load, one of the like comparisons presented associates of the words belonging to the forget category, and one of the like comparisons presented words from the neutral category. Each of the three different comparisons contained one word from either the remember, forget, or neutral category paired with an unrelated word. Participant responses (i.e., "L" or "D") initiated the presentation of the next pair of asterisks.

\section{RECOGNITION TASK}

Following the 18 trials of the ALTM task (i.e., 18 memory loads each followed by a category comparison phase), participants completed the recognition task. During the recognition task, one word was presented at a time in the center of the screen. The participants were instructed to respond "yes" (by pressing the "Y" key) if the word had been presented aurally during any of the memory loads earlier in the experiment and to respond "no" (by pressing the "N" key) if the word had not been heard during the memory loads. Participants were told explicitly to respond "yes" to any word that they had heard during the experiment regardless of the direction to remember or forget that particular item.

The recognition task contained 216 words. Every word from the memory load, both remember (36 words) and forget items (36 words), was included in the recognition task. The task also included distracter items that belonged to the remember ( 18 words) and forget (18 words) categories but were never used during any component of the ALTM task. There were also novel items belonging to the neutral categories (54 words) from the ALTM task, as well as novel items (54 words) belonging to categories never used during the ALTM task. Accuracy was collected for each response. Each response initiated the presentation of the next word.

\section{Procedure}

Upon arrival to the lab, participants read and signed a consent form. They were then seated at one of four sound-dampening computer carrels. Participants were randomly assigned to one of six counter-balanced versions of the experiment. Counter-balancing ensured that each category was used as the remember, forget, and neutral category. The experiment started with a series of instructional slides describing the procedure. The participants were told that there will be a memory load followed by a remember/forget statement and category comparisons. The participants were also informed that their memory of the -remembered items would be tested. The participants were instructed to put on the headphones and their hearing was tested. The hearing test required the participants to type a three-digit number that was presented aurally via the headphones. The participants first com- 
pleted four practice ALTM task trials. Following the ALTM practice, the participants completed a practice recognition task. The practice recognition task did not include any forget items from the practice ALTM trials. Items used for the practice ALTM task and recognition task were not used in any other portion of the experiment. The practice ALTM and recognition trials were identical to the actual trials with the exception of stimuli used. Following the practice phase, participants were informed they were then to complete the actual trials. Each participant then completed 18 actual ALTM task trials. The final phase of the experiment was the actual recognition task. The actual recognition task was preceded by an instructional slide that stated explicitly that participants were to respond yes to any items that were heard during any portion of the experiment including items they were told to forget.

\section{Results}

\section{FACILITATION OF PRIOR COGNITIVE OPERATIONS}

Presented first are the results of the category comparison trials from the ALTM task. For the sake of brevity, only results from the category comparisons that were like (i.e., positive match) trials are presented. This was done as both theory and evidence (e.g., Woltz, 1990; Woltz \& Was, 2006, 2007) indicate that response facilitation is minimal or nonexistent in negative matched comparison trials. In addition, stimuli were not counterbalanced or randomly assigned to positive and negative matched comparisons and this could lead to a confounding of match type and content.

Table 1 presents the means and SDs of reaction and accuracy for the positive matched comparisons by category condition (remember, forget, and neutral). The results indicate possible facilitation in both accuracy and latency. To test for significant facilitation, a dependent measure was calculated by combining RT and accuracy. Because facilitation effects were apparent in both measures a rate correct score (RCS, see Vandierendonck, 2018) was calculated by dividing the participants' accuracy for each category comparison condition by the sum of the RTs for each trial in that condition (both correct and incorrect). The RCS equals the proportion of correct responses divided by response latency divided by 60.000 and is interpreted as the number of correct responses per minute. This measure is an index of response speed adjusted for errors and has been demonstrated to be appropriate for incorporating and combining meaningful variance from both latency and accuracy (e.g., Vandierendonck, 2018, Was, 2010; Woltz \& Was, 2006, 2007). Figure 2 presents the average RCS by each trial condition. To test for facilitation, a repeated-measures ANOVA was conducted with two orthogonal contrasts. The first contrast compared the combined average of the remember and forget category comparison conditions with that of the neutral (nonfacilitated) comparison condition to test for an overall effect of memory load processing. Participants were faster in responding to the remember and forget than to neutral category comparisons, $F(1,35)=13.31, \mathrm{MSe}=79.04, p=.001, \eta^{2}=0.27$. This result suggests response facilitation for responses to categories represented in the memory load component compared to neutral categories. Of greater importance to the current investigation, RCS was greater for forget category comparisons compared to neutral category comparisons, $F(1$, $35)=10.88, \mathrm{MSe}=103.58, p=.002, \eta^{2}=0.24$. This result suggests response facilitation for the comparisons of the categories participants were instructed to forget compared to neutral categories.

\section{RECOGNITION}

Table 2 displays the means and SDs for recognition accuracy for each condition. Of the 216 words presented in the recognition task 72 (33\%) were from the memory load, $36(16 \%)$ were associated with remember and forget categories but not seen in the ALTM task, 54 (25\%) were associates of the neutral categories and 54 (25\%) were novel words. It was thought necessary to balance the category element in the recognition task. Put differently, the memory load categories (remember and forget combined) were each represented by six words (four memory load items and two associates). As there were 18 ALTM task trials, this created 108 recognition trials related to the memory loads. Therefore, 54 neutral category associates and 54 novel items were chosen as stimuli in the recognition task. This not only allowed for an even number of items related and unrelated to the memory loads, it also allowed to test for false alarms on associates of the memory load and neutral categories, which may be related to residual activation. However, this may have created a confound as participants might have recognized the ma-

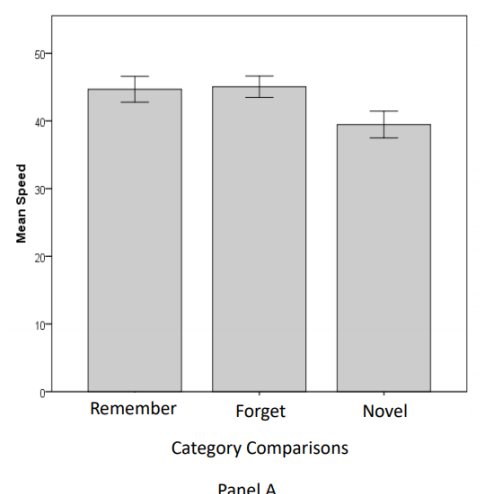

Panel A

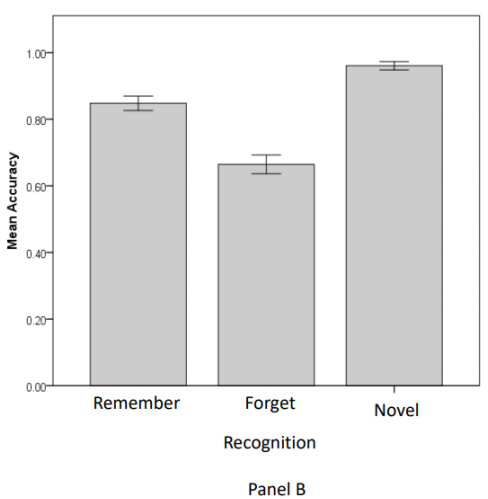

Panel B

FIGURE 2.

Mean RCS for comparisons and recognition accuracy by each trial condition in Experiment 1. 
TABLE 1.

Means and Standard Deviations of Category Comparison ACcuracy and Reaction Time by Category Type from Experiment 1

\begin{tabular}{lcccc}
\hline & \multicolumn{2}{r}{ Accuracy } & \multicolumn{2}{c}{ Reaction time (ms) } \\
\hline \multicolumn{1}{c}{ Comparison Type } & $M$ & $S D$ & $M$ & $S D$ \\
\hline Remember & .89 & .11 & 1265 & 325 \\
Forget & .91 & .07 & 1262 & 304 \\
Novel & .84 & .10 & 1394 & 485 \\
Overall & .88 & .06 & 1307 & 347 \\
\hline
\end{tabular}

jority of words were not heard during the memory load and defaulted to an "answer no" strategy. Put differently, there were twice as many "No" response items than "Yes" response items. This could promote a response bias inflating accuracy for "No" response items. However, the dependent measure for the test of our hypothesis, that facilitation of procedural memory is not dependent on continued availability of declarative memory, is the difference in recognition accuracy between forget and remember items from the memory load. Items from these conditions both require a Yes response and therefore would be equally affected by a No response bias.

Due to the possible response bias, three analyses were conducted to determine if participants were performing better than chance. Typically, such an analysis would use a value of .50 as for dichotomous responses with equally distributed correct responses. As the responses in our experiment were not evenly distributed (.67 for No and .33 for Yes), 2 one-sample $t$ tests using .67 and .33 as cutoff values for overall recognition accuracy were conducted. Both tests were significant, $t(35)$ $=21.72, p<.001, d=7.34,95 \% \mathrm{CI}[.19, .23]$, and $t(35)=56.86, p<.001$, $d=19.22,95 \%$ CI $[.53, .57]$, respectively. This indicates that participants were not performing at chance. Additionally, we calculated criterion $C$ as a method of detecting potential response bias. Due to the uneven number of correct Yes and No responses, the optimal criterion $C$ value would be .36. The observed criterion $C$ was .56. The larger value of the observed criterion compared to the optimal criterion suggests that participants were more conservative (i.e., were more likely to respond No) in their responses.

Participant recognition accuracy was significantly better for remember $(M=.76, S D=.16)$ than for forget items $(M=.58, S D=.16)$, $t(35)=9.86, p<.001, d=1.13,95 \%$ CI $[.14, .21]$. Put differently, participants were less accurate at recognizing memory load items that they

TABLE 2.

Means and Standard Deviations of Recognition Accuracy for Experiment 1

\begin{tabular}{lll}
\hline \multicolumn{1}{c}{ Stimuli } & \multicolumn{2}{c}{ Accuracy } \\
\hline Remember & $M$ & $S D$ \\
Remember distractor & .76 & .16 \\
Forget & .88 & .18 \\
Forget distractor & .58 & .16 \\
Neutral & .88 & .14 \\
Novel & .93 & .12 \\
Overall & .96 & .12 \\
\hline
\end{tabular}

were instructed to forget compared to items they were instructed to remember (see Figure 2B).

Although not a main focus of the experiment, we also examined whether processing of the memory load exemplars and categories would lead to false alarms on the remember and forget associate exemplars. Due to the pertinent interest in memory load items, false alarms on category associates may indicate residual activation related to the memory load categories. To test for false alarms, recognition accuracy was aggregated for the forget and remember distractors and the same was done for the neutral and novel items. A dependent samples $t$ test found that participants tended to false alarm on the distractor items more often than the novel and neutral items, $t(35)=5.73, p<.001, d$ $=.46,95 \%$ CI [.04 .08].

\section{Discussion}

In their Experiment 2, Woltz and Was (2006) found that when participants were told to ignore a category, responses to comparisons containing exemplars of that category were facilitated to a significantly greater magnitude than to the category they were required to remember. However, participants were not required to recall items from the ignored category. Pertinent to this investigation was participant recognition of the memory load items they were instructed to forget. It has important implications regarding the mechanisms underlying long-term semantic priming.

The results of Experiment 1 in the current study indicate that participant recognition of the forget category exemplars was less accurate in relation to recognition of the remember exemplars. Importantly, there was significant facilitation of the category comparisons containing associates of the forget memory load exemplars, but less accurate recognition of the memory load items from those same categories in the recognition task. One explanation is that the mechanisms responsible for successful completion of the two tasks are different. Put differently, the act of forgetting certain items (i.e., not maintaining an available representation of the items) only impacts a specific mechanism for recognition (e.g., semantic priming, episodic memory), and a separate mechanism allows access to an item specifically related to encoding for the comparison phase (e.g., facilitation of the cognitive operation of categorization).

To summarize, participants' responses to the category comparisons containing associates of memory load exemplars they were instructed to forget were significantly facilitated, but participants' recognition of the memory load items from those same categories was less accurate compared to the -remembered exemplars. There was also significant facilitation of the comparisons related to items they were instructed to remember and greater accuracy of recognition for remember items. Therefore, a forget instruction led to category-specific facilitation during category comparisons but item-specific impairment during the recognition task. However, a remember instruction also led to category-specific improvement on category comparisons and significantly better item-specific recognition.

Although the results of Experiment 1 are promising, we recognize the imbalance in correct responses in the recognition tasks was prob- 
lematic. We also recognize the need for replication when results are notable, though somewhat expected. We conducted Experiment 2 to serve two purposes: First, to test whether the results of the Experiment 1 are robust, and second, to control for the possible response bias in the Experiment 1 recognition task.

\section{EXPERIMENT 2}

The method of Experiment 2 was identical to those of Experiment 1 with one exception; the recognition task had an equal number of yes and no correct responses. Having an equal number of Yes and No responses during the recognition task was meant to control for potential response bias. The recognition task in Experiment 2 used a total of 108 items. Items included 27 items related to the remember category (18 old and 9 new), 27 related to the forget category (18 old and 9 new), and 54 neutral category items ( 18 old and 36 new).

\section{Participants}

We used the same a priori power estimation as in Experiment 1 and planned to recruit 32-36 participants. Due to the manner in which the lab scheduling system recruits participants, a total of 45 undergraduate education majors participated and were compensated with course credit. None of the participants in Experiment 2 participated in Experiments 1 or 3 . Eight of the participants were males and 36 were females, one participant did not report their gender. Their median age was 19 years old (range $=15$ years).

\section{Results}

Two repeated-measures ANOVAs were conducted to examine the results of Experiment 2. The first was used to measure the facilitation effects during the category-comparison phase of the experiment. The second was used to measure the recognition accuracy between stimulus types during the recognition task.

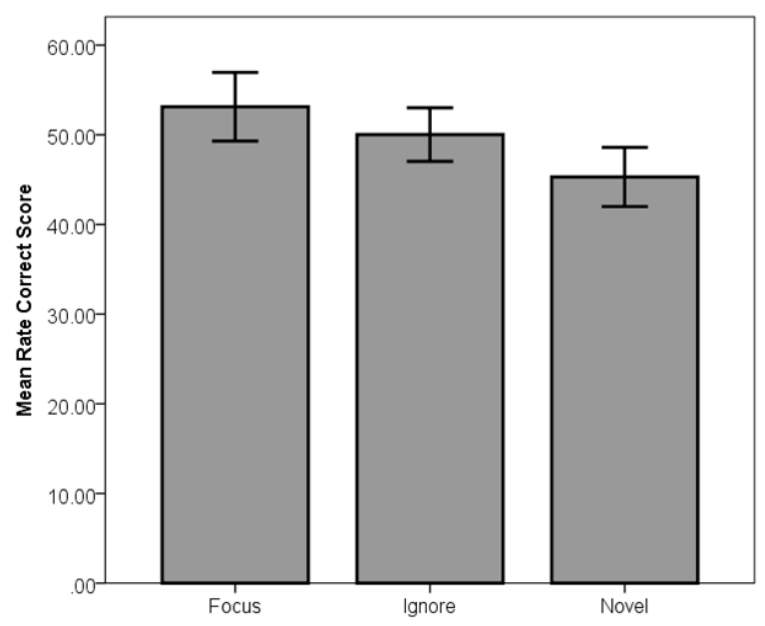

FIGURE 3.

Mean RCS for comparisons by each trial condition in Experiment 2.

\begin{tabular}{|c|c|c|c|c|}
\hline \multirow[b]{2}{*}{ Comparison Type } & \multicolumn{2}{|c|}{ Accuracy } & \multicolumn{2}{|c|}{ Reaction time (ms) } \\
\hline & $M$ & $S D$ & $M$ & $S D$ \\
\hline Remember & .92 & .07 & 1173 & 327 \\
\hline Forget & .88 & .09 & 1205 & 362 \\
\hline Novel & .88 & .09 & 1229 & 346 \\
\hline Overall & .89 & .08 & 1202 & 345 \\
\hline
\end{tabular}

Descriptive statistics for the category comparisons are presented in Table 3. To test the facilitation effects during category comparisons, two orthogonal contrasts were conducted. The first contrast compared the combined average RCS of remember and forget category comparisons to the neutral category comparisons. The purpose of this contrast was to determine if there was a difference between memory load categories and neutral categories. Indeed, there was an effect for memory load categories versus neutral categories, $F(1,44)=9.26$, MSe $=89.07, p=.004, \eta^{2}=0.17$. The second contrast was meant to measure the difference in RCS between the forget and neutral categories. Unlike in Experiment 1, the difference between the RCS of forget category comparisons and neutral category comparisons was not significant, $F(1,44)=2.20, \mathrm{MSe}=112.41, p=.15, \eta^{2}=0.08$, though the effect was in the expected direction. A graphical depiction of the comparison results is shown in Figure 3.

To test for effects in the recognition data, a repeated-measures ANOVA was conducted. The recognition accuracy means and standard deviations for the remember, forget, and novel items are displayed Table 4. A significant main effect for recognition type was found, $F(2$, $90)=79.42, \mathrm{MSe}=.02, p<.001, \eta^{2}=0.64$. Pairwise comparisons revealed significant differences between each of the item types in the recognition tasks, $p<.001$.

\section{Discussion}

Experiment 2 was designed to address the possibility that an imbalance of Yes and No correct responses in Experiment 1 may have led to a bias in participant recognition performance. As such, the recognition task in Experiment 2 used balanced Yes and No correct responses (i.e., of the 108 items in the recognition task, 54 were previously presented category exemplars and 54 were not). The recognition results of Experiment 2 replicated those of Experiment 1, suggesting that the potential for response bias did not meaningfully alter the original findings. As expected, there was a significant difference between each of the three levels of the recognition condition and the highest to lowest recognition accuracy belonged to the novel, remember, and forget categories, respectively. Notably, the forget items were recognized as previously presented less often than the remember items and the criterion $C$ values showed a lack of response bias, hence replicating the findings of Experiment 1.

Of particular interest was the replication of the long-term semantic priming effect during category comparisons in Experiment 1, specifically the test of facilitation differences of memory load categories (remember and forget) over neutral categories. As is in previous research and Experiment 1, RCS for category comparisons trials of memory load 
TABLE 4.

Means and Standard Deviations of Recognition Accuracy for Experiment 2

\begin{tabular}{lll}
\hline \multicolumn{1}{c}{ Stimuli } & \multicolumn{2}{c}{ Accuracy } \\
\hline Remember & $M$ & $S D$ \\
Forget & .84 & .15 \\
Novel & .66 & .19 \\
Overall & .96 & .08 \\
\hline
\end{tabular}

items was significantly greater than RCS for neutral category comparisons. Furthermore, no statistically significant difference was found when comparing for the RCS of category processing between the remember and forget categories. However, unlike in Experiment 1, there was no statistically significant difference in RCS between the forget categories and the neutral categories. The pattern of results between Experiments 1 and 2 are similar though not identical.

Important aspects of the results of Experiment 2 replicate those of Experiment 1. Category comparisons of exemplars presented in the memory load were facilitated relative to neutral categories and recognition for forget memory load items was reduced compared to both neutral and remember items. Although RCS was not significantly greater for the forget compared to neutral category comparisons, it was in the hypothesized direction. This is the first instance of this effect not being significant. We address this in greater detail in the General Discussion section.

The results of Experiments 1 and 2 suggest that the mechanism responsible for the facilitation of the category-specific operation during the comparisons is independent of the mechanism responsible for performance during the recognition task. However, an alternative explanation for the recognition task results is that participants used strategies during the ALTM portion of the task to recall the remember exemplars-for example, explicit rehearsal-that allowed for the more accurate recognition of those stimuli. As the evidence presented thus far is only applicable to the memory load items (i.e., category exemplars) and not to the category labels, it is possible that the facilitation effect found for the forget category comparisons might be explained by encoding of the category labels. Put differently, when participants are instructed to forget a category, they must encode that category and it is possible that an explicit trace of the category remains, or residual activation of the category allows for the availability of the category during the comparison task. Therefore, rather than testing recognition for the memory load exemplars, in Experiment 3, we tested recognition for the category labels. Just as in Experiments 1 and 2, in Experiment 3, participants were told to remember exemplars from one category for later recall and to forget the other exemplars, but during the recognition task, participants were asked if they had encountered the specific categories. Therefore, in Experiment 3, if the remember category label is recognized more frequently as having been previously encountered than the forget category, this would suggest that the recognition results of Experiments land 2 cannot be entirely explained by the rehearsal of the remember exemplars. Experiment 3 was conducted to measure recognition for the category labels themselves to test this hypothesis.

\section{EXPERIMENT 3}

While the facilitation of cognitive operations has been replicated several times, there remains a question as to how reliant it is upon availability of relevant declarative elements. The results of Experiments 1 and 2 suggest that the demonstrated facilitation effects do not rely wholly upon a continued availability of memory load items. In the previous experiments, differential recognition was found for remember and forget items following equivalent facilitation effects. Despite the suggested independence of the mechanisms responsible for categoryspecific comparisons and item-specific recognition in Experiments 1 and 2, there remains the possibility that the remember category exemplars are continually available throughout the category comparison portion of the task via a mechanism such as explicit rehearsal. We hypothesized that a clear advantage would exist in recognition of the remember category labels over the forget category labels, suggesting that the facilitation of exemplar comparisons is not due to a strategy to maintain activation of the specific remember category exemplars. If the facilitation effects should replicate those of previous experiments and the effect cannot be explained by the influence of category labels, then the proposed distinctiveness of the two mechanisms will have been demonstrated between availability of declarative memory elements (i.e., individual memory load items as well as the broader categories themselves) and procedural memory elements (i.e., the relevant cognitive operations) of the ALTM task. Put differently, if our prediction is correct, this would provide further evidence that the mechanism underlying facilitation of exemplar comparisons is distinct from those underlying the recognition task.

\section{Participants}

The participants in Experiment 3 were 51 undergraduate education majors who did not participate in Experiments 1 or 2. Participation in Experiment 3 was compensated with course credit. Six of the participants were males and 45 were females. Their median age was 20 years old (range $=5$ ).

\section{Results}

Similar to Experiments 1 and 2, two repeated-measures ANOVAs were used to analyze the data from Experiment 3 . The first was used to measure the facilitation effect during the category-comparison phase of the experiment. The second was used to measure the recognition accuracy for categories between stimulus types.

Table 5 lists accuracy and latency for each comparison type. To examine the effects of stimulus type during the category comparison phase of the experiment, a repeated-measures ANOVA was conducted with two orthogonal contrasts. The first contrast compared the combined average RCS of remember and forget category comparisons to the neutral category comparisons. The purpose of this contrast was to determine if there was a difference between memory load categories and neutral categories. Indeed, there was an effect for memory load categories versus neutral categories, $F(1,50)=27.96$, $\mathrm{MSe}=71.90, p<$ $.001, \eta^{2}=0.36$. The second contrast was meant to measure the differ- 
TABLE 5.

Means and Standard Deviations of Category Comparison Accuracy and Reaction Time by Category Type Experiment 3

\begin{tabular}{lcccc}
\hline & \multicolumn{2}{r}{ Accuracy } & \multicolumn{2}{c}{ Reaction time (ms) } \\
\hline \multicolumn{1}{c}{ Comparison Type } & $M$ & $S D$ & $M$ & $S D$ \\
\hline Remember & .91 & .08 & 1096 & 295 \\
Forget & .89 & .08 & 1121 & 297 \\
Novel & .84 & .08 & 1196 & 366 \\
Overall & .88 & .08 & 1307 & 347 \\
\hline
\end{tabular}

ence in RCS between the forget and neutral categories. $F(1,50)=12.87$, MSe $=88.39, p=.001, \eta^{2}=.21$.

Descriptive statistics for the recognition phase are listed in Table 6. A repeated-measures ANOVA was conducted to analyze the recognition data. A significant main effect was detected between stimuli types of remember, $M=.83(S D=.02)$, forget, $M=.62(S D=.03)$, and novel, $M=.83(S D=.02), F(2,100)=22.75, \mathrm{MSe}=.03, p<.001, \eta^{2}=0.31$. Pairwise comparisons showed that recognition accuracy was significantly worse for the forget categories compared to the remember, $t(50)$ $=-7.31, p<.001$, and novel categories, $t(50)=-4.56, p<.001$. There was no statistically significant difference between the remember and novel categories, $t(50)=.052, p=.96$.

\section{Discussion}

Following Experiments 1 and 2, the question remained as to the separateness between the mechanisms underlying the facilitation observed during the category comparison trials and those underlying the maintained availability of declarative memory elements. Specifically, Experiment 3 was designed to rule out the possibility that the category labels presented in the memory load would remain active and subsequently contribute to the category comparison facilitation effects found in Experiments 1 and 2. The results showed that participants recognized the forget categories less often than the remember categories and suggest that the facilitation was not dependent upon availability of the category labels. Thus, Experiment 3 provides further evidence that the cognitive operation of exemplar categorization is distinct from that of availability of the category representation.

\section{GENERAL DISCUSSION}

\section{Continually Cumulating Meta-Analyses}

A continually cumulating meta-analysis (Braver et al., 2014) was conducted to clarify effects from the three experiments. The metaanalyses were conducted using a spreadsheet utility designed by Sibley (2008). Descriptions of the relative magnitude of Cohen's $d$ effect sizes were adopted from Cohen (1988).

We examined the relationship between the forget and neutral category comparisons across the three experiments. Experiments 1 and 3 found significant facilitation of forget category comparisons over neutral category comparisons, but Experiment 2 did not. A meta-analysis of the RCS difference between forget category comparisons and neutral category comparisons revealed pooled Cohen's $d=.36, p=.004 ; Q=$ $1.48, p=.48$, and $I^{2}=.0$. As a series, the three experiments demonstrate a significant facilitation of forget comparisons over neutral comparisons.

Woltz and Was $(2006,2007)$ proposed that the long-term semantic priming effects found in the ATLM task are predominately due to the facilitation of prior cognitive operations. The general goal of the current investigation was to investigate whether the mechanisms underlying the facilitation of category comparisons are separate and distinct from the mechanisms responsible for maintaining availability of relevant declarative memory elements. Three experiments were conducted in pursuit of this goal. Each of the three experiments, utilizing an experimental paradigm adapted from directed forgetting research, demonstrated a relative lack of recognition for items participants were instructed to forget compared to remembered items. In support of the explanation of the ALTM task effects through a facilitation of prior cognitive operations, the categorization of those forget items that is assumed to occur following the forget instruction led to facilitation in forget category comparison trials. The same items that participants struggled to recognize were critical in producing the demonstrated long-term sematic priming. Therefore, it is suspected that the mechanisms underlying the exemplar comparison facilitation and the recognition task are qualitatively different. The evidence in this investigation supports the notion that the demonstrated facilitation relies on the strength of the cognitive operations of categorization whereas the recognition task relies on either episodic declarative memory for specific items or categories. If recognition relies on available or retrievable declarative memory elements, recognition failure in the presence of facilitation for categorization provides evidence that the facilitation is less dependent or even separate and distinct from continued availability of the same declarative elements.

The facilitation of prior cognitive operations in category comparison facilitation in the ALTM task has interesting implications for explanations of complex cognition. First, the results of the current study support the hypothesis that some long-term semantic priming effects are in part due to memory for prior cognitive operations.

Second, measures of individual differences in the facilitation of cognitive operations task have been shown to account for unique variance in complex cognitions, including reasoning and comprehension, above and beyond that of WM capacity (Was et al., 2012; Was \& Woltz, 2007; Zamary et al., 2019). More commonly, individual differences in complex cognition are predicted from measures of WM capacity (Conway et al., 2003). However, the empirical limits of WM capacity

\section{TABLE 6.}

Means and Standard Deviations of Recognition Accuracy for Experiment 3

\begin{tabular}{lll}
\hline \multicolumn{1}{c}{ Stimuli } & \multicolumn{2}{c}{ Accuracy } \\
\hline Remember & $M$ & $S D$ \\
Forget & .83 & .15 \\
Novel & .62 & .23 \\
Overall & .83 & .18 \\
\hline
\end{tabular}


struggle to account for the large amounts of information often required for complex cognition (Baddeley, 2012; Cowan, 1988, 2001; Ericsson \& Kintsch, 1995). Therefore, it seems clear that WM is not alone in accounting for complex cognition. In fact, several theorists suggest that WM must have efficient access to LTM (e.g., Baddeley, 2012; Ericsson \& Kintsch, 1995). In this regard, the strengthening of specific cognitive operations, as demonstrated in the ALTM task, may be a way in which WM efficiently interacts with long-term memory. For example, the declarative elements in a comprehension task activate relevant procedures, and those procedures are then relatively more efficient upon subsequent use without having to maintain the declarative elements that initially instantiated them. The newly facilitated procedures then allow for efficient processing of relevant information without overloading WM.

The results of the current study suggest that the mechanisms underlying long-term priming are to some degree independent from those used for maintaining or retrieving relevant declarative memory elements. The results suggest that the mechanisms underlying longterm semantic priming are to some degree independent from those used for maintaining or retrieving relevant declarative memory elements. This suggests that continued facilitation of cognitive operations does not necessitate continued maintenance of the declarative elements required to instantiate the procedures. As such, participants may have both explicit awareness and implicit learning or memory of the task procedure, as described by Seger (1994), without having continuing awareness of the specific items (cf. Schacter, 1992).

An alternative explanation for the results is based on Oberauer's (2009) procedural WM perspective. Oberauer described the bridge as activated procedures from long-term memory. The procedures are activated by relevant declarative elements in the region of direct access. A single declarative element activates multiple procedures, then task characteristics (e.g., goals) determine which procedure is applied. Facilitation is defined by using a procedure in the bridge rather than having to search LTM for an appropriate procedure. During the ALTM task, the procedure "determine if $\mathrm{X}$ is a $\mathrm{Y}$ " is retrieved from LTM into the bridge by the declarative elements (i.e., exemplars) in the memory load of the task. Later, when making category comparisons, "determine if $\mathrm{X}$ is a $\mathrm{Y}$ " remains in the bridge, but the original declarative elements do not necessarily remain in the region of direct access. Indeed, our results indicate it is not necessary for those elements to remain for the procedure to still be in the bridge.

One possible limitation of the current study is that we did not use an implicit measure of recognition following the ALTM task. Basden et al (1993) and Paller (1990) reported that item-method directing forgetting effects occur for explicit memory tests but not for implicit tests. It is possible that although participants did not have an explicit memory of the forget items, the items were still in an available state and that an implicit memory test (e.g., lexical decisions) would have captured the residual availability. Be that as it may, it is clear that retrieval of the forget items was impaired, yet the operation of exemplar categorization was facilitated for those items.
The current study has potentially important implications for models of human cognition. It seems appropriate to have access to relevant long term-memory when engaging in a complex cognitive task such as comprehension. The current study clearly indicates that the facilitation of category comparisons is not dependent on the active maintenance of declarative memory elements.

\section{ACKNOWLEDGEMENTS}

The views expressed in this article are those of the authors and do not necessarily reflect the official policy or position of the Department of the Navy, Department of Defense, nor the U.S. Government.

\section{REFERENCES}

Anderson, J. R. (1993). Rules of the mind. Psychology Press.

Baddeley, A. (2012). Working memory: theories, models, and controversies. Annual Review of Psychology, 63, 1-29. https://doi. org/10.1146/annurev-psych-120710-100422 16لس

Basden, B. H., Basden, D. R., \& Gargano, G. J. (1993). Directed forgetting in implicit and explicit memory tests: A comparison of methods. Journal of Experimental Psychology: Learning, Memory, and Cognition, 19, 603. https://doi.org/10.1037/0278-7393.19.3.603 المالسلس Bastin, C., Feyers, D., Majerus, S., Balteau, E., Degueldre, C., Luxen, A. Pierre Maquet, Salmon, E., \& Collette, F. (2012). The neural substrates of memory suppression: A FMRI exploration of directed forgetting. PloS One, 7, e29905. https://doi.org/10.1371/journal. pone.0029905 المالسلس

Braver, S. L., Thoemmes, F. J., \& Rosenthal, R. (2014). Continuously cumulating meta-analysis and replicability. Perspectives on Psychological Science, 9, 333-342. https://doi.org/10.1177/1745691614529796 الس Becker, S., Moscovitch, M., Behrmann, M., \& Joordens, S. (1997). Long-term semantic priming: A computational account and empirical evidence. Journal of Experimental Psychology: Learning, Memory, \& Cognition, 23, 1059-1082. https://doi.org/10.1037/02787393.23.5.1059 الس الس

Cohen, J. (1988). Statistical power analysis for the behavioral sciences. Lawrence Earlbaum Associates.

Collins, A. M., \& Loftus, E. F. (1975). A spreading-activation theory of semantic processing. Psychological Review, 82, 407. https://doi. org/10.1037/0033-295X.82.6.407 المالسلسل

Conway, A. R., Kane, M. J., \& Engle, R. W. (2003). Working memory capacity and its relation to general intelligence. Trends in Cognitive

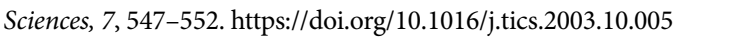

Cowan, N. (1988). Evolving conceptions of memory storage, selective attention, and their mutual constraints within the human information-processing system. Psychological Bulletin, 104, 163-191.

Cowan, N. (1995). Attention and memory: An integrated framework. Oxford University Press.

Cowan, N. (1999). An embedded-processes model of working memory. In A. Miyake \& P. Shah (Eds.), Models of working memory: Mechanisms of active maintenance and executive control (p. 62-101). Cambridge University Press 
Cowan, N. (2001). Metatheory of storage capacity limits. Behavioral and Brain Sciences, 24, 154-176. Iالسلس

Dosher, B. A., \& Rosedale, G. (1989). Integrated retrieval cues as a mechanism for priming in retrieval from memory. Journal of Experimental Psychology: General, 118, 191-211. https://doi. org/10.1037/0096-3445.118.2.191 السلس

Ericsson, K. A., \& Kintsch, W. (1995). Long-term working memory. Psychological Review, 102, 211-245. https://doi.org/10.1037/0033295X.102.2.211 سلس

Golding, J. M. (2005). Directed forgetting tasks in cognitive research. In A. Wenzel \& D. C. Rubin (Eds.), Cognitive methods and their application to clinical research (p. 177-196). American Psychological Association.

Hughes, A. D., \& Whittlesea, B. W. (2003). Long-term semantic transfer: An overlapping-operations account. Memory \& Cognition, 31, 401-411. https://doi.org/10.3758/BF03194398 سلسلس

Hourihan, K. L., \& Taylor, T. L. (2006). Cease remembering: control processes in directed forgetting. Journal of Experimental Psychology: Human Perception and Performance, 32, 1354-1365. https://doi. org/10.1037/0096-1523.32.6.1354 سلس

Joordens, S., \& Becker, S. (1997). The long and short of semantic priming effects in lexical decision. Journal of Experimental Psychology: Learning, Memory, \& Cognition, 23, 1083-1105. https://doi. org/10.1037/0278-7393.23.5.1083 المالسلس

Just, M. A., \& Carpenter, P. A. (1992). A capacity theory of comprehension: Individual differences in working memory. Psychological Review 99, 122-149. https://doi.org/10.1037/0033-295X.99.1.122 المالسلسلا

Masson, M. E. (1995). A distributed memory model of semantic priming. Journal of Experimental Psychology: Learning, Memory, and Cognition, 21, 3-23. https://doi.org/10.1037/0278-7393.21.1.3 سلس

McNamara, T. P. (1992). Priming and constraints it places on theories of memory and retrieval. Psychological Review, 99, 650-662. السلسلسل|

McNamara, T. P. (2005). Semantic priming: Perspectives from memory and word recognition. Psychology Press.

Oberauer, K. (2002). Access to information in working memory: Exploring the focus of attention. Journal of Experimental Psychology: Learning, Memory, and Cognition, 28, 411-421. https://doi. org/10.1037/0278-7393.28.3.411 السلسلس

Oberauer, K. (2009). Design for a working memory. Psychology of Learning and Motivation, 51, 45-100. https://doi.org/10.1016/ S0079-7421(09)51002-X Wلس

Paller, K. A. (1990). Recall and stem-completion priming have different electrophysiological correlates and are modified differentially by directed forgetting. Journal of Experimental Psychology: Learning, Memory, and Cognition, 16, 1021-1032. https://doi. org/10.1037/0278-7393.16.6.1021 15

Quillian, M. R. (1967). Word concepts: A theory and simulation of some basic semantic capabilities. Behavioral Science, 12, 410-430. https://doi.org/10.1002/bs.3830120511 لسلسلس

Ratcliff, R., \& McKoon, G. (1988). A retrieval theory of priming in memory. Psychological Review, 95, 385-408. السلسلس

Seger, C. A. (1994). Implicit learning. Psychological Bulletin, 115,
163-196. https://doi.org/10.1037/0033-2909.115.2.163 1سلس

Schacter, D. L. (1992). Consciousness and awareness in memory and amnesia: Critical issues. In A. D. Miller \& M. D. Rugg (Eds.), The neuropsychology of consciousness (p. 179-200). Academic Press.

Schneider, W., Eschman, A., \& Zuccolotto, A. (2002). E-prime computer software and manual. Psychology Software Tools Inc.

Sibley, C. G. (2008). Utilities for examining simple meta-analytic averages [Computer software]. University of Auckland.

Taylor, T. L. (2005). Inhibition of return following instructions to remember and forget. The Quarterly Journal of Experimental Psychology Section A, 58, 613-629. https://doi.org/10.1080/02724980443000115 المالسلسلة

Taylor, T. L., Cutmore, L., \& Pries, L. (2018). Item-method directed forgetting: Effects at retrieval? Acta Psychologica, 183, 116-123. https:// doi.org/10.1016/j.actpsy.2017.12.004 الس الس

Taylor, T. L., \& Fawcett, J. M. (2011). Larger IOR effects following forget than following remember instructions depend on exogenous attentional withdrawal and target localization. Attention, Perception, \& Psychophysics, 73, 1790-1814. https://doi.org/10.3758/s13414-0110146-2 2 |لسلس

Vandierendonck, A. (2018). Further tests of the utility of integrated speed-accuracy measures in task switching. Journal of Cognition, 1, 1-16. https://doi.org/10.5334/joc.6 السلسلسلس

Was, C. A. (2010). The persistence of content-specific memory operations: Priming effects following a 24-h delay. Psychonomic Bulletin \& Review, 17, 362-368. https://doi.org/10.3758/PBR.17.3.362 المالسلسل

Was, C. A., Dunlosky, J., Bailey, H., \& Rawson, K. A. (2012). The unique contributions of the facilitation of procedural memory and working memory to individual differences in intelligence. Acta Psychologica,

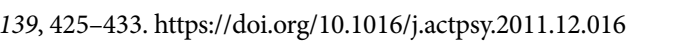

Was, C. A., \& Woltz, D. J. (2007). Reexamining the relationship between working memory and comprehension: The role of available long-term memory. Journal of Memory and Language, 56, 86-102. https://doi.org/10.1016/j.jml.2006.07.008 المالسلس

Woltz, D. J. (1990). Repetition of semantic comparisons: Temporary and persistent priming effects. Journal of Experimental Psychology: Learning, Memory, and Cognition, 16, 392-403. https://doi.

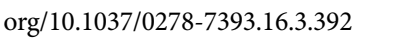

Woltz, D. J., \& Was, C. A. (2006). Availability of related long-term memory during and after attention focus in working memory. Memory \& Cognition, 34, 668-684. https://doi.org/10.3758/BF03193587 المالسلسلة

Woltz, D. J., \& Was, C. A. (2007). Available but unattended conceptual information in working memory: Temporarily active semantic content or persistent memory for prior operations?. Journal of Experimental Psychology: Learning, Memory, and Cognition, 33, 155-168. https://doi.org/10.1037/0278-7393.33.1.155 سلس

Zamary, A., Rawson, K. A., \& Was, C. A. (2019). Do complex span and content-embedded working memory tasks predict unique variance in inductive reasoning? Behavior Research Methods, 51, 2546-2558. https://doi.org/10.3758/s13428-018-1104-x المبلمالسالس

RECEIVED 16.09.2020 | ACCEPTED 15.05.2021 